\title{
Gamma-Ray Spectrometry of Humans at the University of Utah
}

Ray D. Lloyd, Charles W. Mays, and David H. Taysum

Radiobiological Laboratory, University of Utah, Salt Lake City, UT 84112, USA

Radiat. Environ. Biophys. 16, 157-175 (1979)

The first equation shown in Fig. 10b, page 173, should have been:

$$
k=0.0636 e^{-1,242 E} .
$$

